\title{
VOLUME INCREASING ISOMETRIC DEFORMATIONS OF CONVEX POLYHEDRA
}

\author{
DAVID D. BLEECKER
}

\begin{abstract}
In this paper, we prove that the surface of a generic convex polyhedron in $E^{3}$ can be isometrically deformed so as to enclose greater volume. Our deformed surfaces have at least seven times more faces than the original surface. They are extrinsically nonconvex, but have the same intrinsic geometry (e.g., area, angle deficits, etc.). Roughly put, the deformations are obtained by simultaneously delivering karate chops to the edges of the polyhedron. While the edges implode, to preserve the intrinsic geometry, portions of the faces move outward, leading to a net increase of volume. The regular tetrahedron can be isometrically deformed to enclose over $37.7 \%$ more volume, while the cube, octahedron, dodecahedron, and icosahedron enjoy increases over $21.8 \%, 11.5 \%, 9.3 \%$, and $3.6 \%$, respectively. Many questions have arisen. In particular, what is the nature of an isometric embedding, if any, of an abstract surface which encloses the maximum volume? We propose that the term "sandbag" be used to describe such an embedding. What kinds of singularities can they have? Are they unique up to Euclidean motions?
\end{abstract}

\section{Introduction}

A deformation of a surface $S$ in Euclidean 3-space $E^{3}$ is a continuous map $h: S \times[0, \varepsilon] \longrightarrow E^{3}$, such that for $h_{t}(\cdot):=h(\cdot, t)$, we have that $h_{0}$ is the inclusion of $S$ in $E^{3}$. The deformation $h$ is an isometric deformation if the length of any rectifiable curve $\gamma$ in $S$ is constant under the deformation; i.e., $L\left(h_{t} \circ \gamma\right)=L(\gamma)$, for all $t \in[0, \varepsilon]$. Thus, the intrinsic geometry (in particular, the area and Gaussian curvature measure of $S$ ) is preserved under an isometric deformation. In our main results, the surface $S$ will be a generic convex polyhedral surface with triangular faces, such that each pair of faces with a common edge meet at a dihedral angle strictly less than $\pi$. However, we also consider the cube and dodecahedron. It seems likely that the assumption of triangular faces is only a technicality and probably can be dropped. For the specific isometric deformation $h$ that we will construct, for $t>0, h_{t}(S)$ will also be a polyhedral surface, but with 7 times as many true faces

Received August 1, 1994. 


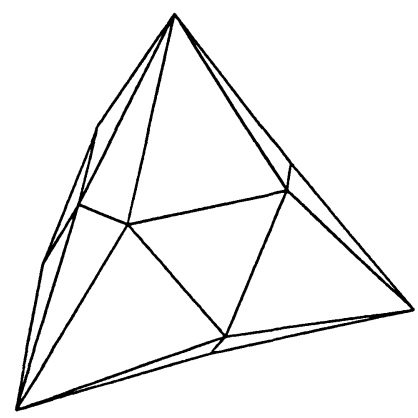

Figure 1

as $S$ in the generic triangular case, 54 faces in the cubical case, and 132 faces for the dodecahedral case. For $t>0, h_{t}(S)$ will not be convex, since by a theorem of Aleksandrov [2], which generalizes Cauchy's theorem ([3], [7], [13]), there are no isometric deformations within the class of convex surfaces, which are not induced by motions of $E^{3}$. The most significant property of our isometric deformation is that $h_{t}(S)$ encloses more volume in $E^{3}$ than $S$ does, for sufficiently small $t>0$. In other words, the guaranteed ([1],[6],[10]) convex isometric embedding of a generic intrinsically convex abstract polyhedral surface does not maximize the enclosed volume functional on the space of all isometric embeddings of the surface in $E^{3}$. What sort of isometric embedding, if any, does maximize the enclosed volume? This is apparently completely unknown, even for a regular tetrahedral or a cubical surface. We will find that upon applying the isometric deformation $h_{t}$ to a regular tetrahedron that one can increase the enclosed volume by more than $37.7 \%$, whereas we obtain more modest increases for the other platonic surfaces, which are given in the table in the last section.

Before getting into the details of the construction of the deformation, a picture of a stage of the deformation as it is applied to a regular tetrahedron is shown in Figure 1. The result might be intuitively described by saying that each of the edges of the original regular tetrahedron is imploded at the midpoint by a karate chop. One might think that this would decrease the enclosed volume. However in the process, a triangular portion of each face is translated outward from the center, thereby producing a net increase in the volume enclosed, for sufficiently mild karate chops. The bulk of the paper is spent proving this assertion for a generic convex polyhedral surface with triangular faces.

\section{Isometric deformations of a triangle}

Let $T$ be a triangular region in $E^{3}$ with angles $\alpha_{1}, \alpha_{2}$ and $\alpha_{3}$ at the 


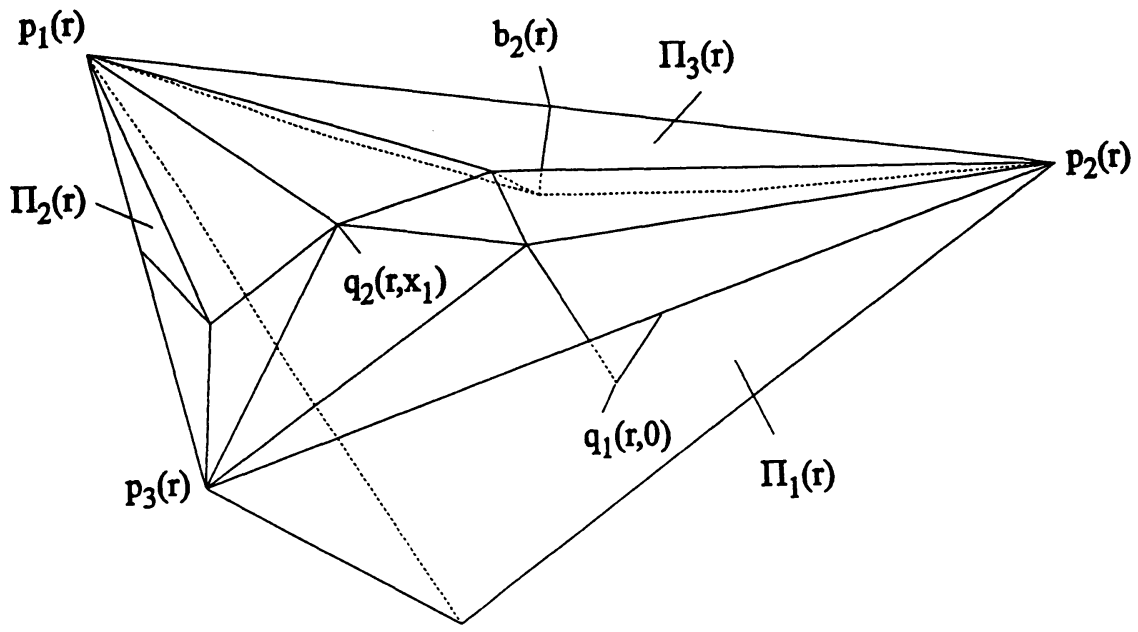

FIGURE 2

vertices $p_{1}, p_{2}$ and $p_{3}$, respectively. Let $\mathbf{L}_{1}, \mathbf{L}_{2}$ and $\mathbf{L}_{3}$ be the sides of $T$ opposite $p_{1}, p_{2}$ and $p_{3}$, and let their lengths be $L_{1}, L_{2}$ and $L_{3}$. Denote the midpoints of $\mathbf{L}_{1} \mathbf{L}_{2}$ and $\mathbf{L}_{3}$, by $b_{1}, b_{2}$ and $b_{3}$. Let $\Pi_{1}, \Pi_{2}$ and $\Pi_{3}$ be closed half-planes with edges including the sides $\mathbf{L}_{1}, \mathbf{L}_{2}$ and $\mathbf{L}_{3}$, and making acute angles $\frac{\pi}{2}-\beta_{1}, \frac{\pi}{2}-\beta_{2}$ and $\frac{\pi}{2}-\beta_{3}$ with $T$, respectively. We assume that $\Pi_{1} \Pi_{2}$ and $\Pi_{3}$ are on the same side of the plane containing $T$. Eventually we will take $\Pi_{1}, \Pi_{2}$ and $\Pi_{3}$ to be the planes which bisect the dihedral angles formed by $T$ and the faces adjacent to $T$ in some convex polyhedral surface in $E^{3}$.

We arbitrarily select an origin $O$ in $E^{3}$, and for $r \in(0,1]$, let $D_{r}$ be a contraction of $E^{3}$ about $O$, which decreases distances by a factor of $r$. We denote the image of $T$ under $D_{r}$ by $T(r)$. Similarly, let the images of $p_{i}, \mathbf{L}_{i}, b_{i}$ and $\Pi_{i}(i=1,2,3)$ under $D_{r}$, be denoted by $p_{i}(r), b_{i}(r), \mathbf{L}_{i}(r)$ and $\Pi_{i}(r)$. Refer to Figure 2. Since $r \in(0,1]$, there are unique points $q_{i}(r) \in \Pi_{i}(r)$, such that $d\left(q_{i}(r), p_{j}(r)\right)=d\left(q_{i}(r), p_{k}(r)\right)=\frac{1}{2} L_{i}$, for all distinct $i, j, k$, where $d(u, v)$ denotes the Euclidean distance between points $u$ and $v$ in $E^{3}$. For $x_{i} \geq 0$, let $q_{i}\left(r, x_{i}\right)$ be the point which is at distance $x_{i}$ along the ray which issues orthogonally from $\Pi_{i}(r)$ at $q_{i}(r)$ toward $T(r)$; note that $q_{i}(r, 0)=q_{i}(r)$.

Let $b_{i}\left(r, x_{i}\right)$ (not illustrated) be the point in $T(r)$ which is at distance $x_{i}$ along the perpendicular bisector ray of $\mathbf{L}_{i}(r)$ which issues from $b_{i}(r)$ into $T(r)$. We denote an arbitrary cyclic permutation of $(1,2,3)$ by $(i, j, k)$. For any $(i, j, k)$, let

$$
f_{i}\left(r, x_{j}, x_{k}\right):=d\left(q_{j}\left(r, x_{j}\right), q_{k}\left(r, x_{k}\right)\right)^{2}-d\left(b_{j}\left(1, x_{j}\right), b_{k}\left(1, x_{k}\right)\right)^{2},
$$

Note that $f_{i}(1,0,0)=0$. If, for some $r \in(0,1]$, there are simultaneous solutions $x_{1}, x_{2}, x_{3}$ of the 3 equations $f_{i}\left(r, x_{j}, x_{k}\right)=0$, then we have the 


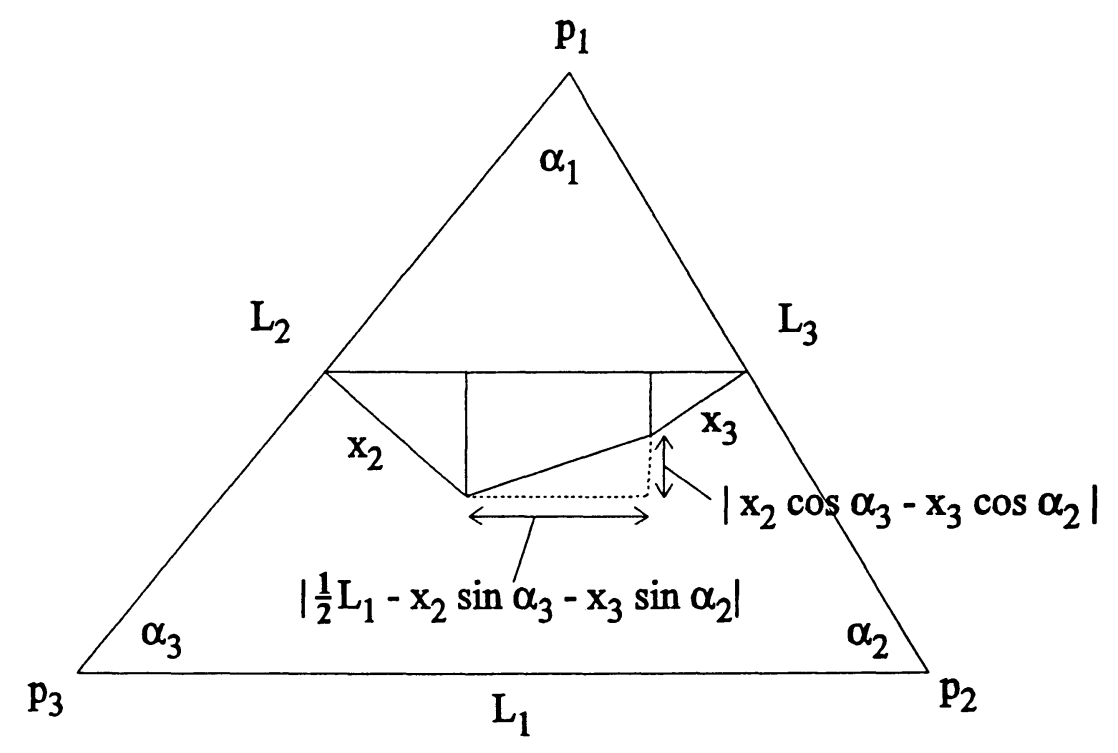

Figure 3

following 10 congruencies of triangles:

$$
\begin{aligned}
\Delta\left(q_{1}\left(r, x_{1}\right), q_{2}\left(r, x_{2}\right), q_{3}\left(r, x_{3}\right)\right) \equiv \Delta\left(b_{1}\left(1, x_{1}\right), b_{2}\left(1, x_{2}\right), b_{3}\left(1, x_{3}\right)\right), \\
\Delta\left(p_{i}(r), q_{j}\left(r, x_{i}\right), q_{k}\left(r, x_{k}\right)\right) \equiv \Delta\left(p_{i}, b_{j}\left(1, x_{j}\right), b_{k}\left(1, x_{k}\right)\right), \\
\quad \text { for all }(i, j, k) \\
\Delta\left(p_{i}(r), q_{j}(r), q_{j}\left(r, x_{j}\right) \equiv \Delta\left(p_{i}, b_{j}(1,0), b_{j}\left(1, x_{j}\right)\right), \text { for all } i \neq j .\right.
\end{aligned}
$$

Thus the union, say $U(r)$, of the 10 triangles on the left is a polyhedral surface that is intrinsically (i.e., except for folding) the same as the union $T$ of the 10 triangles on the right. If we can find simultaneous solutions $x_{i}$ depending continuously on all $r$ close to 1 , then we can obtain an isometric deformation of $T$, by moving each point of $T$ to its image in $U(r)$ under the congruencies (2). To this end we will produce an explicit expression for $f_{i}\left(x_{j}, x_{k}, r\right)=d\left(q_{j}\left(r, x_{j}\right), q_{k}\left(r, x_{k}\right)\right)^{2}-d\left(b_{j}\left(1, x_{j}\right), b_{k}\left(1, x_{k}\right)\right)^{2}$.

Note that $d\left(b_{j}\left(1, x_{j}\right), b_{k}\left(1, x_{k}\right)\right)^{2}$ is readily found from Figure 3 above in which the hypotenuse of the right triangle with dashed legs is $d\left(b_{2}\left(1, x_{2}\right), b_{3}\left(1, x_{3}\right)\right)$. 


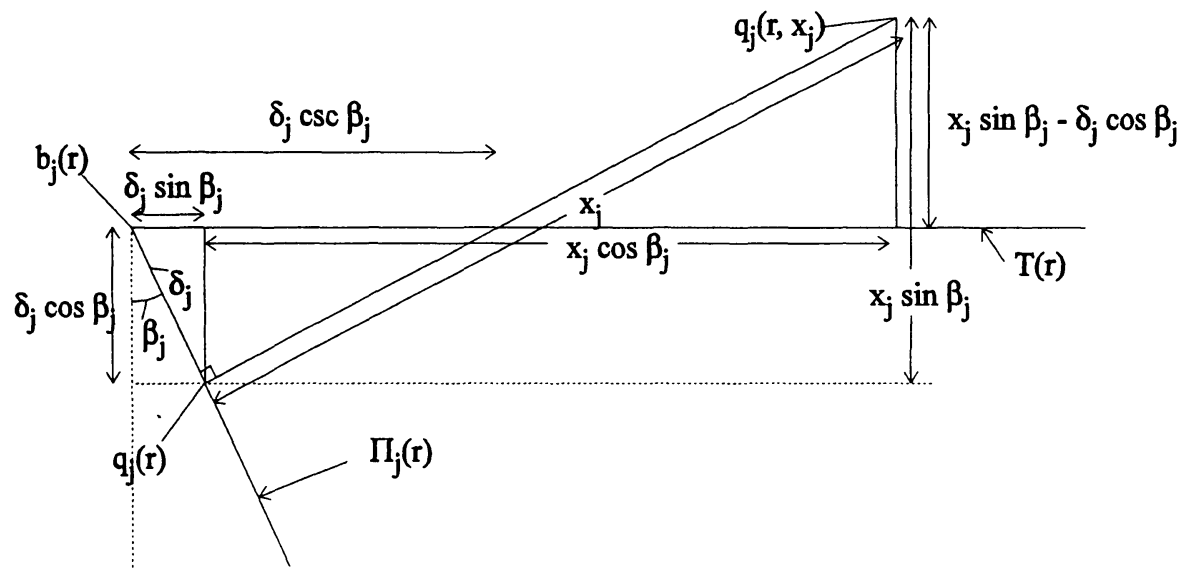

Figure 4

Thus, for all $(i, j, k)$, we have

$$
\begin{aligned}
d\left(b_{j}\left(1, x_{j}\right), b_{k}\left(1, x_{k}\right)\right)^{2}= & \left(\frac{1}{2} L_{i}-x_{j} \sin \alpha_{k}-x_{k} \sin \alpha_{j}\right)^{2} \\
& +\left(x_{j} \cos \alpha_{k}-x_{k} \cos \alpha_{j}\right)^{2} \\
= & x_{j}^{2}+x_{k}^{2}+2\left(\sin \alpha_{k} \sin \alpha_{j}-\cos \alpha_{k} \cos \alpha_{j}\right) x_{j} x_{k} \\
& -L_{i}\left(x_{j} \sin \alpha_{k}+x_{k} \sin \alpha_{j}\right)+\frac{1}{4} L_{i}^{2} \\
= & x_{j}^{2}+x_{k}^{2}-2 \cos \left(\alpha_{k}+\alpha_{j}\right) x_{j} x_{k} \\
& -L_{i}\left(x_{j} \sin \alpha_{k}+x_{k} \sin \alpha_{j}\right)+\frac{1}{4} L_{i}^{2} \\
= & x_{j}^{2}+x_{k}^{2}+2 \cos \alpha_{i} x_{j} x_{k} \\
& -L_{i}\left(x_{j} \sin \alpha_{k}+x_{k} \sin \alpha_{j}\right)+\frac{1}{4} L_{i}^{2} .
\end{aligned}
$$

We compute $d\left(q_{j}\left(r, x_{j}\right), q_{k}\left(r, x_{k}\right)\right)^{2}$ as follows. The points $p_{i}(r), b_{j}(r)$ and $q_{j}(r)$ form a right triangle with right angle at $b_{j}(r)$. Therefore,

$$
d\left(p_{i}(r), b_{j}(r)\right)^{2}+d\left(b_{j}(r), q_{j}(r)\right)^{2}=d\left(p_{i}(r), q_{j}(r)\right)^{2}=\left(\frac{1}{2} L_{j}\right)^{2} .
$$

Since $d\left(p_{i}(r), b_{j}(r)\right)=\frac{1}{2} r L_{j}$, we have $d\left(b_{j}(r), q_{j}(r)\right)^{2}=\left(\frac{1}{2} L_{j}\right)^{2}\left(1-r^{2}\right)$. Thus setting $r=\cos t$ for $0 \leq t<\frac{\pi}{2}$, we have $d\left(b_{j}(r), q_{j}(r)\right)=\frac{1}{2} L_{j} \sin t$. In Figure $4, d\left(b_{j}(r), q_{j}(r)\right)=\frac{1}{2} L_{j} \sin t$ is denoted by $\delta_{j}$. In this figure, we are viewing a cross section by a plane, which is orthogonal to the edge $\mathbf{L}_{j}(r)$ at its midpoint $b_{j}(r)$. Hence, we are viewing $T(r)$ and $\Pi_{j}(r)$ edge on. The orthogonal projection of $q_{j}\left(r, x_{j}\right)$ onto the plane of $T(r)$ is at a distance of $\delta_{j} \sin \beta_{j}+x_{j} \cos \beta_{j}$ along the perpendicular bisector ray of $\mathbf{L}_{j}(r)$ issuing from $b_{j}(r)$. So, this projection is $b_{j}\left(r, \delta_{j} \sin \beta_{j}+x_{j} \cos \beta_{j}\right)$. 
Let $x_{j}^{\prime}=\delta_{j} \sin \beta_{j}+x_{j} \cos \beta_{j}$. If we apply (3) to the points $b_{j}\left(r, x_{j}^{\prime}\right)$ and $b_{k}\left(r, x_{k}^{\prime}\right)$ in $T(r)$, we obtain

$$
\begin{aligned}
d\left(b_{j}\left(r, x_{j}^{\prime}\right), b_{j}\left(r, x_{k}^{\prime}\right)\right)^{2}= & {x_{j}^{\prime}}^{2}+{x_{k}^{\prime}}^{2}+2 \cos \alpha_{i} x_{j}^{\prime} x_{k}^{\prime} \\
& -r L_{i}\left(x_{j}^{\prime} \sin \alpha_{k}+x_{k}^{\prime} \sin \alpha_{j}\right)+\frac{1}{4} r^{2} L_{i}^{2} .
\end{aligned}
$$

This is the square, say $H_{j k}^{2}$, of the length of the orthogonal projection onto the plane of $T(r)$ of the segment $q_{j}\left(r, x_{j}\right) q_{k}\left(r, x_{k}\right)$. To get $d\left(q_{j}\left(r, x_{j}\right), q_{k}\left(r, x_{k}\right)\right)^{2}$, we must add to $L_{j k}^{2}$ the square, say $V_{j k}^{2}$, of the difference in distances of $q_{j}\left(r, x_{j}\right)$ and $q_{k}\left(r, x_{k}\right)$ from the plane of $T(r)$. From Figure 4, it is seen that

$$
V_{j k}^{2}=\left(x_{j} \sin \beta_{j}-\delta_{j} \cos \beta_{j}-\left(x_{k} \sin \beta_{k}-\delta_{k} \cos \beta_{k}\right)\right)^{2} .
$$

Therefore, with $r=\cos t$ and recalling that $\delta_{j}=\frac{1}{2} L_{j} \sin t$, we have

$$
\begin{aligned}
f_{i}(r, & \left.x_{j}, x_{k}\right) \\
= & d\left(q_{j}\left(r, x_{j}\right), q_{k}\left(r, x_{k}\right)\right)^{2}-d\left(b_{j}\left(1, x_{j}\right), b_{k}\left(1, x_{k}\right)\right)^{2} \\
= & H_{j k}^{2}+V_{j k}^{2}-d\left(b_{j}\left(1, x_{j}\right), b_{k}\left(1, x_{k}\right)\right)^{2} \\
= & \left(\frac{1}{2} L_{j} \sin t \sin \beta_{j}+x_{j} \cos \beta_{j}\right)^{2} \\
& +\left(\frac{1}{2} L_{k} \sin t \sin \beta_{k}+x_{k} \cos \beta_{k}\right)^{2} \\
& +2 \cos \alpha_{i}\left(\frac{1}{2} L_{j} \sin t \sin \beta_{j}+x_{j} \cos \beta_{j}\right) \\
& \quad \cdot\left(\frac{1}{2} L_{k} \sin t \sin \beta_{k}+x_{k} \cos \beta_{k}\right) \\
& -L_{i} \cos t\left(\left(\frac{1}{2} L_{j} \sin t \sin \beta_{j}+x_{j} \cos \beta_{j}\right) \sin \alpha_{k}\right. \\
& \left.+\left(\frac{1}{2} L_{k} \sin t \sin \beta_{k}+x_{k} \cos \beta_{k}\right) \sin \alpha_{j}\right)+\frac{1}{4} \cos ^{2} t L_{i}^{2} \\
& +\left(x_{j} \sin \beta_{j}-\frac{1}{2} L_{j} \sin t \cos \beta_{j}-x_{k} \sin \beta_{k}+\frac{1}{2} L_{k} \sin t \cos \beta_{k}\right)^{2} \\
& -\left(x_{j}^{2}+x_{k}^{2}+2 \cos \alpha_{i} x_{j} x_{k}-L_{i}\left(x_{j} \sin \alpha_{k}+x_{k} \sin \alpha_{j}\right)+\frac{1}{4} L_{i}^{2}\right)
\end{aligned}
$$




$$
\begin{aligned}
& =2\left(\cos \alpha_{i}\left(\cos \beta_{j} \cos \beta_{k}-1\right)-\sin \beta_{j} \sin \beta_{k}\right) x_{j} x_{k} \\
& +\left(L_{k} \sin t\left(\cos \alpha_{i} \cos \beta_{j} \sin \beta_{k}+\sin \beta_{j} \cos \beta_{k}\right)\right. \\
& \left.\quad+L_{i} \sin \alpha_{k}\left(1-\cos t \cos \beta_{j}\right)\right) x_{j} \\
& +\left(L_{j} \sin t\left(\cos \alpha_{i} \sin \beta_{j} \cos \beta_{k}+\sin \beta_{k} \cos \beta_{j}\right)\right. \\
& \left.\quad+L_{i} \sin \alpha_{j}\left(1-\cos t \cos \beta_{k}\right)\right) x_{k} \\
& +\frac{1}{4} \sin ^{2} t\left(2 L_{j} L_{k}\left(\cos \alpha_{i} \sin \beta_{j} \sin \beta_{k}-\cos \beta_{j} \cos \beta_{k}\right)\right. \\
& \left.\quad+L_{j}^{2}+L_{k}^{2}-L_{i}^{2}\right) \\
& -L_{i} \frac{1}{2} \sin t \cos t\left(L_{j} \sin \beta_{j} \sin \alpha_{k}+L_{k} \sin \beta_{k} \sin \alpha_{j}\right)
\end{aligned}
$$

Consider the mapping $\mathbf{F}: \mathbb{R}^{1} \times \mathbb{R}^{3} \longrightarrow \mathbb{R}^{3}$ given by $\mathbf{F}:=\left(F_{1}, F_{2}, F_{3}\right)$, where $F_{i}(t, \mathbf{x}):=F_{i}\left(t, x_{1}, x_{2}, x_{3}\right):=f_{i}\left(\cos t, x_{j}, x_{k}\right)$ for a cyclic permutation $(i, j, k)$ of $(1,2,3)$, i.e.,

$$
\begin{aligned}
\mathbf{F}(t, \mathbf{x}) & =\mathbf{F}\left(t, x_{1}, x_{2}, x_{3}\right) \\
& =\left(f_{1}\left(\cos t, x_{2}, x_{3}\right), f_{2}\left(\cos t, x_{3}, x_{1}\right), f_{3}\left(\cos t, x_{1}, x_{2}\right)\right)
\end{aligned}
$$

Note that $\mathbf{F}$ is real-analytic, and $\mathbf{F}(0,0,0,0)=(0,0,0)$. If we can show that $\operatorname{det}\left[\partial_{x_{j}} F_{i}(0, \mathbf{0})\right] \neq 0$, then we may apply the implicit function theorem to deduce that there are neighborhoods $(-\varepsilon, \varepsilon)$ of $0 \in \mathbb{R}$ and $B$ about $0 \in \mathbb{R}^{3}$, such that for $t \in(-\varepsilon, \varepsilon)$, there is a unique $\mathbf{x}(t) \in B$ with $\mathbf{F}(t, \mathbf{x}(t))=\mathbf{0}$. Moreover, $\mathbf{x}:(-\varepsilon, \varepsilon) \longrightarrow B \subseteq \mathbb{R}^{3}$ is a realanalytic curve. For distinct $i, j, k$ we have $\partial_{x_{j}} F_{i}(0,0)=\partial_{x_{j}} f_{i}(1,0,0)=$ $L_{i} \sin \alpha_{k}\left(1-\cos \beta_{j}\right)$. Using $1-\cos \beta_{j}=2 \sin ^{2}\left(\frac{1}{2} \beta_{j}\right)$, it readily follows that

$$
\operatorname{det}\left[\partial_{x_{j}} F_{i}(0,0)\right]=16 \prod_{1 \leq k \leq 3} L_{k} \sin \alpha_{k} \sin ^{2}\left(\frac{1}{2} \beta_{k}\right) \neq 0
$$

We also need $x_{i}(t)>0(i=1,2,3)$ for sufficiently small $t>0$, and for this it suffices to prove that $x_{i}^{\prime}(0)>0$. Taking the $t$ derivative of the equation $\mathbf{F}(t, \mathbf{x}(t))=\mathbf{0}$, we obtain at $t=0,\left(F_{i}\right)_{t}(0, \mathbf{0})+\left(\nabla F_{i}\right)(0, \mathbf{0})$. $\mathbf{x}^{\prime}(0)=0(i=1,2,3)$. Solving these 3 equations in the unknowns $x_{i}^{\prime}(0)$, 
one finally obtains $x_{i}^{\prime}(0)=\frac{1}{2} L_{i} \cot \left(\frac{1}{2} \beta_{i}\right)$. We check this explicitly:

$$
\begin{aligned}
\left(F_{i}\right)_{t}(0, \mathbf{0})+ & \left(\nabla F_{i}\right)(0, \mathbf{0}) \cdot \mathbf{x}^{\prime}(0) \\
= & -L_{i} \frac{1}{2}\left(L_{j} \sin \beta_{j} \sin \alpha_{k}+L_{k} \sin \beta_{k} \sin \alpha_{j}\right) \\
& +L_{i} \sin \alpha_{k}\left(1-\cos \beta_{j}\right) \frac{1}{2} L_{j} \cot \left(\frac{1}{2} \beta_{j}\right) \\
& +L_{i} \sin \alpha_{j}\left(1-\cos \beta_{k}\right) \frac{1}{2} L_{k} \cot \left(\frac{1}{2} \beta_{k}\right) \\
= & 0,
\end{aligned}
$$

since $\cot \left(\frac{1}{2} \beta_{j}\right)=\sin \beta_{j} /\left(1-\cos \beta_{j}\right)$. Thus, as noted before, the solution curve $\mathbf{x}(t)$ gives rise to an isometric deformation of the triangle $T$ via the congruencies (2).

\section{Deforming a generic closed convex surface}

Theorem 1. Let $S$ be an embedded convex polyhedral surface in $E^{3}$ which has triangular faces, with the dihedral angle formed by any pair of adjoining faces strictly less than $\pi$. Then there is an isometric deformation $h: S \times[0, \varepsilon] \longrightarrow E^{3}$, through isometric embeddings, such that for $t>0$ sufficiently small, $v(t)>v(0)$, where $v(t)$ is the volume enclosed by $h_{t}(S)$.

Proof. For each triangle $T$ of $S$, we use the same notation as in Section 2 , and we let $\Pi_{1}, \Pi_{2}$, and $\Pi_{3}$ be the half planes which bisect the dihedral angles formed by $T$ and the three triangles adjacent to $T$. Since the isometric deformations of all of the triangles agree on all of the common edges, we obtain a well defined isometric deformation $h:[0, \varepsilon] \times S \longrightarrow S$ of all of $S$.

By choosing $\varepsilon$ smaller if necessary, we argue that $h_{t}: S \longrightarrow h_{t}(S)$ is an isometric embedding for all $0 \leq t \leq \varepsilon$, so that $v(t)$ is well defined, as follows. Note that deformations of interiors of triangles sharing a common edge lie in different half-spaces. For triangles $T_{1}$ and $T_{2}$ sharing but one common vertex $p$, note that each sufficiently small segment of $T_{1}$ issuing from $p$ into $T_{1}$ is mapped by $h_{t}$ to segment issuing from $h_{t}(p)$ whose direction changes continuously with $t$. Since the directions of the segments of $T_{2}$ issuing from $p$ are bounded away from the directions of segments of $T_{1}$ issuing from $p$, no sufficiently small neighborhood of $h_{t}(p)$ in $h_{t}\left(T_{1}\right)$ will meet a sufficiently small neighborhood of $h_{t}(p)$ in $h_{t}\left(T_{2}\right)$, except at $h_{t}(p)$. Since we have just shown that distinct points which are sufficiently close cannot have the same image under $h_{t}$ for $t$ sufficiently small, the fact that $h_{t}$ is an embedding for $t$ sufficiently small follows easily from the fact that $h_{0}$ is an embedding.

It remains to show that $v(t)>v(0)$, for $t>0$ sufficiently small. We accomplish this by computing the difference, say $n(t)$, between $v(t)$ 


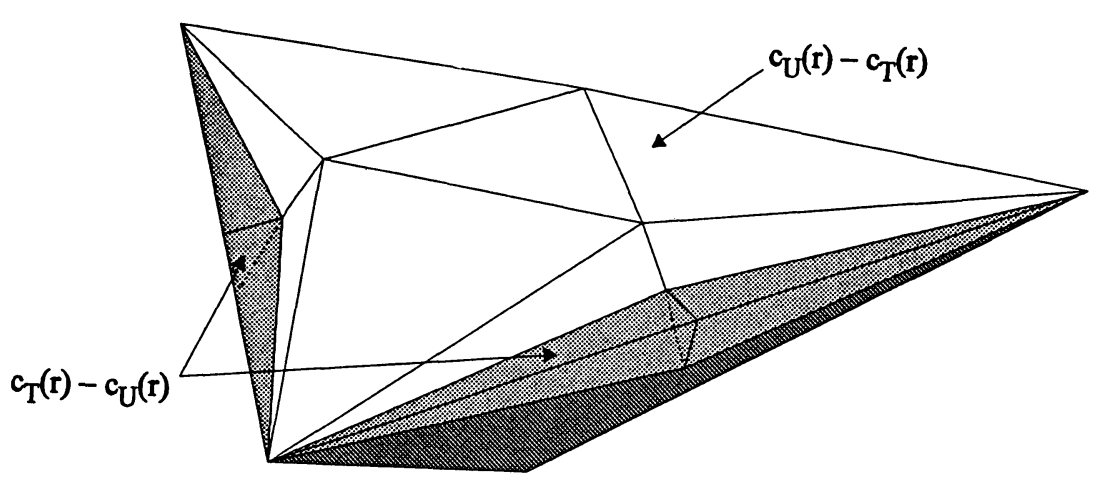

FIGURE 5

and the volume $w(t)$ enclosed by $D_{\cos t}(S)$ for a contraction $D_{\cos t}$ by a factor of $\cos t$ about the arbitrary point $O$ of $E^{3}$. We know that $w(t)=$ $\cos ^{3} t w(0)=w(0)+O\left(t^{2}\right)$. Thus $v(t)=w(t)+n(t)=w(0)+n(t)+O\left(t^{2}\right)$. Consider a fixed triangle $T(r)$, where $r=\cos t$. The planes $\Pi_{1}(r), \Pi_{2}(r)$ and $\Pi_{3}(r)$ meet transversely at some point in $E^{3}$, and divide $E^{3}$ into 8 solid angles, one of which, say $c(r)$, contains $T(r)$. Now $T(r)$ truncates $c(r)$ to form a solid tetrahedron, say $c_{T}(r)$. Moreover, the deformation $U(r)$ of $T$ also cuts off a solid piece of $c(r)$, say $c_{U}(r)$. If $c_{U}(r)-c_{T}(r)$ denotes the set-theoretic difference, then

$$
n(t)=\sum_{T}\left(\operatorname{vol}\left(c_{U}(r)-c_{T}(r)\right)-\operatorname{vol}\left(c_{T}(r)-c_{U}(r)\right)\right),
$$

where the sum ranges over all $T$ comprising $S$. We will show that $\operatorname{vol}\left(c_{T}(\cos t)-c_{U}(\cos t)\right)=O\left(t^{2}\right)$, while $\operatorname{vol}\left(c_{U}(\cos t)-c_{T}(\cos t)\right) \geq K t$ for some $K>0$. For small $t>0, c_{T}(\cos t)-c_{U}(\cos t)$ consists of 3 components each of which is a pair of thin solid tetrahedrons which are joined at a common face, as shown in Figure 5.

Indeed, the common face of one of these pairs is the triangle $\Delta\left(b_{j}(r), q_{j}(r), m_{j}(r)\right)$ in Figure 4 , where $m_{j}(r)$ is the point where the segment $q_{j}(r) q_{j}\left(r, x_{j}(t)\right)$ pierces the interior of $T(r)$. Note that for small $t, q_{j}(r)$ and $q_{j}\left(r, x_{j}(t)\right)$ are on opposite sides of the plane of $T(r)$, since (using $\cot \left(\frac{1}{2} \theta\right) \sin \theta=1+\cos \theta$ )

$$
\begin{aligned}
x_{j}(t) \sin \beta_{j}-\delta_{j}(t) \cos \beta_{j} & =\frac{1}{2} L_{j} t \cot \left(\frac{1}{2} \beta_{j}\right) \sin \beta_{j}-\frac{1}{2} L_{j} t \cos \beta_{j}+O\left(t^{2}\right) \\
& =\frac{1}{2} L_{j} t+O\left(t^{2}\right)>0,
\end{aligned}
$$

for sufficiently small $t$. The volume of $c_{T}(\cos t)-c_{U}(\cos t)$ is easily seen to be $\sum_{j} \frac{1}{3} \delta_{j}(t)^{2} \tan \beta_{j} L_{j}=\frac{1}{12}\left(\sum_{j} \tan \beta_{j} L_{j}^{3}\right) \sin ^{2} t=O\left(t^{2}\right)$. In order 
to estimate $\operatorname{vol}\left(c_{U}(\cos t)-c_{T}(\cos t)\right)$ note (see Figure 5) that there is a center piece of $c_{U}(\cos t)-c_{T}(\cos t)$ consisting of a solid triangular prism which is swept out as the triangle $\Delta\left(q_{1}\left(r, x_{1}(t)\right), q_{2}\left(r, x_{2}(t)\right), q_{3}\left(r, x_{3}(t)\right)\right)$ is orthogonally projected onto $T(r)$. The height of this triangular prism is initially 0 at $t=0$, while the triangular base in $T(r)$ of this triangular prism is initially $\frac{1}{4} A$, where $A$ is the area of $T=T(\cos (0))$. The average height of the prism is $\frac{1}{3} \sum_{j}\left(x_{j}(t) \sin \beta_{j}-\delta_{j}(t) \cos \beta_{j}\right)=\frac{1}{6}\left(L_{1}+L_{2}+L_{3}\right) t+$ $O\left(t^{2}\right)$. Thus, the volume of the prism is $\frac{1}{24} A\left(L_{1}+L_{2}+L_{3}\right) t+O\left(t^{2}\right)$. The remaining part of $c_{U}(\cos t)-c_{T}(\cos t)$ also makes an $O(t)$ contribution to the volume, but already we have $\operatorname{vol}\left(c_{U}(\cos t)-c_{T}(\cos t)\right)>\frac{1}{25} A\left(L_{1}+\right.$ $\left.L_{2}+L_{3}\right) t$ for sufficiently small $t>0$.

Remark. More precisely one can show that

$$
\operatorname{vol}\left(c_{U}(\cos t)-c_{T}(\cos t)\right)=\frac{1}{8} A\left(L_{1}+L_{2}+L_{3}\right) t+O\left(t^{2}\right)
$$

Thus, under the deformation $h$, the enclosed volume of $h_{t}(S)$ increases at an initial rate equal to $\frac{1}{8} \sum_{n} A_{n} P_{n}$, where $A_{n}$ and $P_{n}$ are the area and perimeter of the $n$-th face of $S$, respectively.

\section{The regular convex polyhedra with triangular faces}

In this section we assume that $S$ is a convex polyhedral surface with unit edge lengths and equilateral triangular faces (i.e., a regular tetrahedron, octahedron, icosahedron, and the degenerate case of a double- covered triangle). We will compute the enclosed volume $h_{t}(S)$. Using the previous notation, there are many simplifications in the equations $f_{i}\left(r, x_{j}, x_{k}\right)=0$, under the assumption of equilateral triangular faces. First, it is reasonable to seek solutions in which the $x_{i}$ all have the same value, say $x$. Recall that $f_{i}\left(r, x_{j}, x_{k}\right):=$ $d\left(q_{j}\left(r, x_{j}\right), q_{k}\left(r, x_{k}\right)\right)^{2}-d\left(b_{j}\left(1, x_{j}\right), b_{k}\left(1, x_{k}\right)\right)^{2}$. Certainly, $f_{i}\left(r, x_{j}, x_{k}\right)=$ 0 is equivalent to $d\left(q_{j}\left(r, x_{j}\right), q_{k}\left(r, x_{k}\right)\right)-d\left(b_{j}\left(1, x_{j}\right), b_{k}\left(1, x_{k}\right)\right)=0$. When the $x_{i}$ are equal, the segment $b_{2}\left(1, x_{2}\right) b_{3}\left(1, x_{3}\right)$, which is the hypotenuse of the right triangle in Figure 3, is parallel to $L_{1}$. Hence, using $\sin \alpha_{k}=$ $\frac{1}{2} \sqrt{3}$

$$
d\left(b_{2}\left(1, x_{2}\right), b_{3}\left(1, x_{3}\right)\right)=\frac{1}{2} L_{i}-x_{j} \sin \alpha_{k}-x_{k} \sin \alpha_{j}=\frac{1}{2}-\sqrt{3} x .
$$

Moreover, in the identity $d\left(q_{j}\left(r, x_{j}\right), q_{k}\left(r, x_{k}\right)\right)^{2}=H_{j k}^{2}+V_{j k}^{2}$, we have 


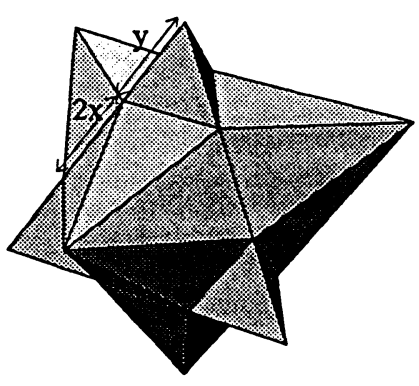

Figure 6

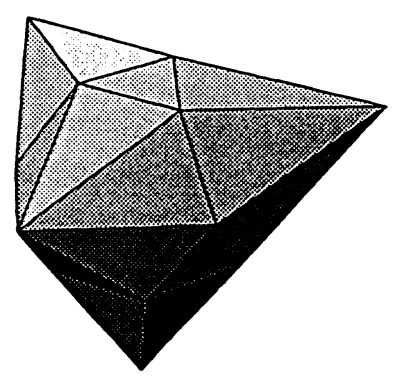

FIGURE 7

$V_{j k}^{2}=0$ under the current assumptions. Thus, referring to Figure 3,

$$
\begin{aligned}
d\left(q_{j}\left(r, x_{j}\right), q_{k}\left(r, x_{k}\right)\right) & =H_{j k}=\frac{1}{2}-\sqrt{3}\left(\delta_{j} \sin \beta_{j}+x_{j} \cos \beta_{j}\right) \\
& =\frac{1}{2}-\sqrt{3}\left(\frac{1}{2} \sin t \sin \beta+x \cos \beta\right),
\end{aligned}
$$

where $\frac{\pi}{2}-\beta$ is half of the interior dihedral angle between the faces. Hence, under the current assumptions, the equations $f_{i}\left(r, x_{j}, x_{k}\right)=0$ are equivalent to

$$
\frac{1}{2}-\sqrt{3}\left(\frac{1}{2} \sin t \sin \beta+x \cos \beta\right)-\left(\frac{1}{2}-\sqrt{3} x\right)=0, \text { or }
$$

$$
x=x(t)=\frac{1}{2} \sin t \frac{\sin \beta}{(1-\cos \beta)}=\frac{1}{2} \sin t \cot \left(\frac{1}{2} \beta\right) .
$$

For the tetrahedron, the cosine of the interior dihedral angle between the faces is $\frac{1}{3}$. Thus, $\frac{1}{3}=\cos (\pi-2 \beta)=-\cos (2 \beta)=-2 \cos ^{2} \beta+$ 1 , or $\cos \beta=\sqrt{1 / 3}$. For the octahedron we obtain $\cos \beta=\sqrt{2 / 3}$, and for the icosahedron we have $\cos \beta=\frac{1}{6}(1+\sqrt{5}) \sqrt{3}$. In each case, $\frac{1}{2} \sin t \cot \left(\frac{1}{2} \beta\right)$ achieves the maximal geometrically allowed value for $x(t)$, namely $\frac{1}{6} \sqrt{3}$, for some $t<\frac{\pi}{2}$. Let

$$
\begin{aligned}
y & =y(t):=d\left(q_{2}(\cos t, x(t)), q_{3}(\cos t, x(t))\right) \\
& =d\left(b_{2}(1, x(t)), b_{3}(1, x(t))\right)=\frac{1}{2}-\sqrt{3} x(t) .
\end{aligned}
$$

For the tetrahedron, we compute the volume enclosed by $h_{t}(S)$ as follows. Consider a solid tetrahedron $T^{\prime}$ of edge length $2 x+2 y$ and 
attach four hexagonal pyramids with alternating base edge lengths of $2 x$ and $y$ to the faces of $T^{\prime}$, as shown in Figure 6 .

The distance from the apex of any pyramid to a closest edge of $T^{\prime}$ is chosen to be $\frac{1}{2}$. Truncate $T^{\prime}$ so that what remains is the solid in Figure 7, say $T^{\prime \prime}$, with triangular faces of side length $y$ along with the triangular faces of the pyramids. The faces of $T^{\prime \prime}$ form the deformation $h_{t}(S)$ (where $\mathrm{x}=\mathrm{x}(\mathrm{t})$ in (14)) of a unit edge tetrahedron $\mathrm{S}$. The volume of $T^{\prime \prime}$ is the volume of the truncated tetrahedron, $\frac{1}{12} \sqrt{s}(2 x+2 y)^{3}-4$. $\frac{1}{12} \sqrt{2} y^{3}$, plus the volumes of the four pyramids. The pyramid base area is $\frac{1}{4} \sqrt{3}(2 y+2 x)^{2}-3 \cdot \frac{1}{4} \sqrt{3} y^{2}=\sqrt{3}\left(x^{2}+2 x y+\frac{1}{4} y^{2}\right)$, while the pyramid height is $\left(\left(\frac{1}{2}\right)^{2}-\left(\frac{1}{6} \sqrt{3}(2 x+2 y)\right)^{2}\right)^{\frac{1}{2}}=\left(\frac{1}{4}-\frac{1}{3}(x+y)^{2}\right)^{\frac{1}{2}}$. Thus the volume of $h_{t}(S)$ in Figure 7 is

$$
\begin{aligned}
V= & \frac{1}{12} \sqrt{2}(2 x+2 y)^{3} \\
& -4 \cdot \frac{1}{12} \cdot \sqrt{2} y^{3}+4 \cdot \frac{1}{3}\left(\frac{1}{4}-\frac{1}{3}(x+y)^{2}\right)^{\frac{1}{2}} \sqrt{3}\left(x^{2}+2 x y+\frac{1}{4} y^{2}\right)
\end{aligned}
$$

$$
=\frac{1}{3} \sqrt{2}\left(2(x+y)^{3}-y^{3}\right)+\frac{1}{3}\left(4 x^{2}+8 x y+y^{2}\right)\left(\frac{3}{4}-(x+y)^{2}\right)^{\frac{1}{2}} .
$$

Since $y=\frac{1}{2}-\sqrt{3} x$, we can find $V$ as a function of $x$. Setting $V^{\prime}(x)=0$, we eventually obtain the sixth-degree equation

$$
\begin{aligned}
& (398208-230272 \sqrt{3}) x^{6}+(400384-230272 \sqrt{3}) x^{5} \\
& +(117392-68032 \sqrt{3}) x^{4}+(5184-3296 \sqrt{3}) x^{3} \\
& +(-1792+1096 \sqrt{3}) x^{2}+(-176+112 \sqrt{3}) x-3=0
\end{aligned}
$$

which defies a solution in terms of radicals. In arriving at this equation, and other analogous sixth-degree equations below, a square root is disposed of by squaring. Thus, extraneous zeros are introduced. However, in the present case (and in each of the cases below) there is only one nonextraneous zero $x_{0}$ in the relevant range $\left[0, \frac{1}{6} \sqrt{3}\right]$. For (17), this zero is given approximately by $x_{0}=0.1354198180198074 \ldots$. One might have conjectured that the maximum volume $V$ is achieved when $y=2 x$, so that the pyramids in Figure 7 have regular bases. However, $y=2 x$ yields $x=1-\frac{1}{2} \sqrt{3}=0.13397459 \ldots$. We have $V\left(x_{0}\right)=0.16230252 \ldots$, while $V\left(1-\frac{1}{2} \sqrt{3}\right)=0.16229774 \ldots$. Since $V\left(x_{0}\right) / V(0)=1.37718257 \ldots$, $V\left(x_{0}\right)$ is over $37.7 \%$ greater than the volume of the undeformed regular tetrahedron. 


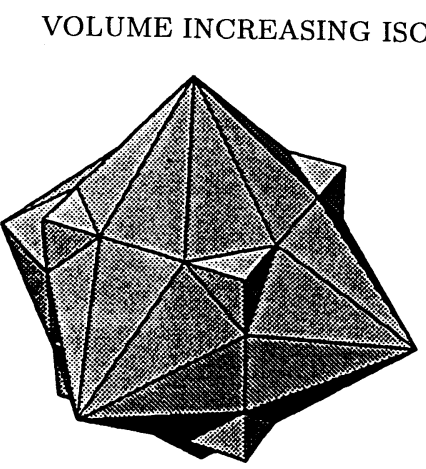

Figure 8

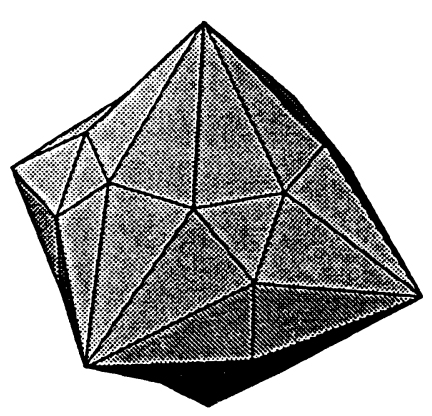

Figure 9

Suppose now that $S$ is an octahedron with edges of unit length. If $x$ and $y$ given by (14) and (15), then we still have $y=\frac{1}{2}-\sqrt{3} x$. In a manner analogous to the case of the tetrahedron, we can decompose the region enclosed by $h_{t}(S)$ into 6 irregular octagonal pyramids with edges of length $y$ and $2 x$ and a truncated cube of original edge length $2 x+\sqrt{2} y$. Figures 8 and 9 show the construction before and after the truncation of the cube. The distance from an apex of a pyramid to a nearest edge of the cube is $\frac{1}{2}$. By adding the volumes of the components, we find that the volume enclosed by $h_{t}(S)$ is given by

$$
\begin{aligned}
V= & (2 x+\sqrt{2} y)^{3}-8 \cdot \frac{1}{6}\left(\frac{y}{\sqrt{2}}\right)^{3} \\
& +2\left((2 x+\sqrt{2} y)^{2}-4 \cdot \frac{1}{2}\left(\frac{y}{\sqrt{2}}\right)^{2}\right)\left(\frac{1}{4}-\left(x+\frac{y}{\sqrt{2}}\right)^{2}\right)^{\frac{1}{2}} \\
= & (2 x+\sqrt{2} y)^{3}-\frac{1}{3} \sqrt{2} y^{3} \\
& +2\left(4 x^{2}+4 \sqrt{2} x y+y^{2}\right)\left(\frac{1}{4}-x^{2}-\sqrt{2} x y-\frac{1}{2} y^{2}\right)^{\frac{1}{2}} .
\end{aligned}
$$

A computation shows that $V^{\prime}(x)=0$ implies

$$
\begin{aligned}
& (1253184 \sqrt{6}-3069696) x^{6}+(1627648 \sqrt{3}-1993344 \sqrt{2}) x^{5} \\
& +(366096 \sqrt{6}-896816) x^{4}+(63904 \sqrt{3}-78336 \sqrt{2}) x^{3} \\
& +(940 \sqrt{6}-2280) x^{2}+(328 \sqrt{2}-264 \sqrt{3}) x+21-9 \sqrt{6}=0 .
\end{aligned}
$$

Again there is only one root $x_{0}$ in the relevant range, which is approximately given by $x_{0}=0.1354140138298575 \ldots$. This first differs in the sixth place from the $x_{0}$ for the tetrahedron. Now $V\left(x_{0}\right)=0.52599693 \ldots$, $V(0)=\frac{1}{3} \sqrt{2}$ and $V\left(x_{0}\right) / V(0)=1.11580800 \ldots$. Thus, the maximum percentage as is increase in the volume for our deformation of the octahedron is about $11.6 \%$ compared to $37.7 \%$ for a tetrahedron. 


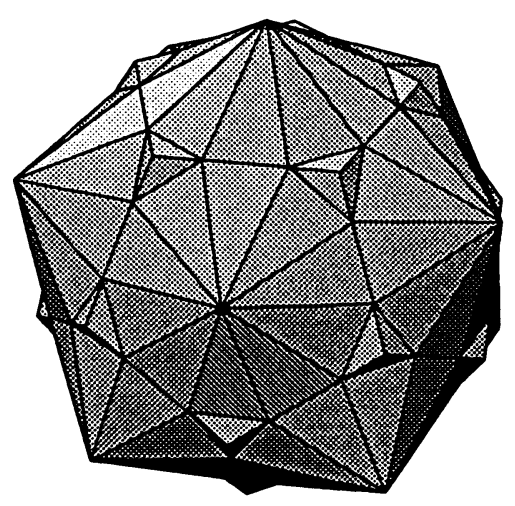

FIGURE 10

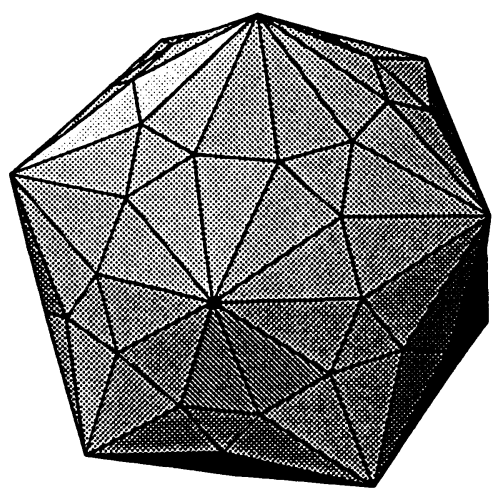

FIGURE 11

For the icosahedron, we compute the volume of the region enclosed by $h_{t}(S)$ by decomposing the region, as in Figures 10 and 11, into 12 pyramids with irregular ten-sided bases with edge lengths $2 x$ and $y$, and a truncated dodecahedron with twenty equilateral triangular faces of edge length $y$. The volume enclosed by $h_{t}(S)$ is then

$$
\begin{aligned}
V= & \frac{1}{4}(7 \sqrt{5}+15)(2 x+y(\sqrt{5}-1))^{3}-\frac{5}{6}(3-\sqrt{5}) y^{3} \\
+ & \left(-4 x^{2}(\sqrt{5}+2)-4 x y(\sqrt{5}+3)-2 y^{2}(\sqrt{5}+1)+\sqrt{5}\right)^{\frac{1}{2}} \\
& 2(9+4 \sqrt{5})^{\frac{1}{4}}\left[x^{2} \sqrt{5}+x y(5-\sqrt{5})\right. \\
& \left.-y^{2}\left(\frac{5}{4}\left(\frac{9-4 \sqrt{5}}{9+4 \sqrt{5}}\right)^{\frac{1}{4}}+\frac{5}{2}-\sqrt{5} \frac{3}{2}\right)\right] .
\end{aligned}
$$

While $V^{\prime}(x)=0$ again implies that $x$ is a zero of a sixth-degree polynomial, we could not simplify the coefficients of the polynomial so that it would fit on a single page. However, there is only one relevant zero in $\left[0, \frac{1}{6} \sqrt{3}\right]$ and it is given approximately by $x_{0}=0.1354116696972618 \ldots$. We have $V(0)=\frac{5}{12}(3+\sqrt{5}), V\left(x_{0}\right)=2.26091586 \ldots$ and $V\left(x_{0}\right) / V(0)=$ $1.03631161 \ldots$. Thus, under the deformation, there is a maximum gain in volume of only about $3.6 \%$ in the case of the icosahedron.

There is an abstract polyhedral surface which consists of two equilateral triangular regions with unit edge length joined together along their boundary, but without identifying interior points. The convex realization of this surface in $E^{3}$ is simply a pair of coincident triangles, known as a double-covered triangle. It encloses no volume and is not an embedding. We can still apply our deformation to the double-covered 


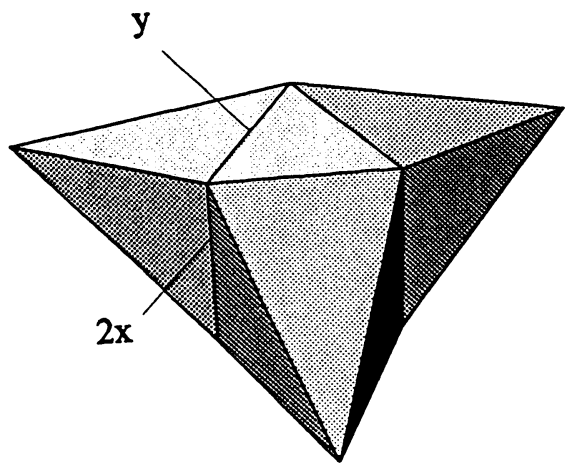

FIGURE 12

triangle, say $S$, to produce a nonconvex embedding of the abstract surface, enclosing a positive volume. For $S$, the interior dihedral angles are zero, and hence the angle $\beta$ is $\frac{\pi}{2}$. Thus, $x(t)=\frac{1}{2} \cot \left(\frac{1}{2} \beta\right) \sin t=\frac{1}{2} \sin t$, and as before, $y=\frac{1}{2}-\sqrt{3} x$. The solid enclosed by the deformed surface $h_{t}(S)$ consists of three square pyramids attached to the $2 x$ by $y$ rectangular faces of an equilateral prism, as is shown in Figure 12.

The volume of this solid is $V=\frac{1}{2} \sqrt{3} y^{2} x+x y\left(1-y^{2}\right)^{\frac{1}{2}}$. Here $V^{\prime}(x)=0$ implies

$$
\begin{aligned}
36288 x^{6}-28224 \sqrt{3} x^{5} & +12144 x^{4}+2400 \sqrt{3} x^{3} \\
& -1116 x^{2}-4 \sqrt{3} x+9=0 .
\end{aligned}
$$

There is but one relevant zero $x_{0}=0.1354446662193181 \ldots$, and $V\left(x_{0}\right)=$ $0.04292061 \ldots$, which is a big improvement over $V(0)=0$.

The cube and dodecahedron

Although the proof of Theorem 1 breaks down for polyhedra with nontriangular faces, we can define analogous volume increasing isometric deformations for the cube and the dodecahedron, due to their symmetry. To produce the deformation for the unit cube, start with a regular octahedron of edge length $2 x+2 y$, where $y=\sqrt{2}\left(\frac{1}{2}-x\right)$ and $0 \leq x \leq \frac{1}{2}$. As in Figure 13, we attach hexagonal pyramids to these faces, so that the distance from apex of a pyramid to a nearest edge of the octahedron is $\frac{1}{2}$. As in Figure 14, truncate the octahedron, so that the 6 new square faces have edge length $y$. The 8 original faces of the octahedron are truncated to generally irregular hexagons of edge lengths $2 x$ and $y$.

Since $y=\sqrt{2}\left(\frac{1}{2}-x\right)$, the bounding surface of the resulting solid is intrinsically isometric to the surface of a cube. The volume of the solid is the volume of the truncated octahedron plus the total volume of the 8 hexagonal pyramids, namely 


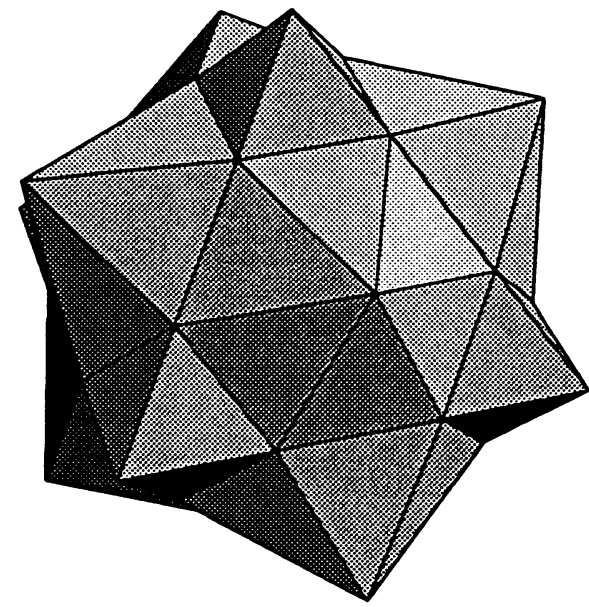

FIGURE 13

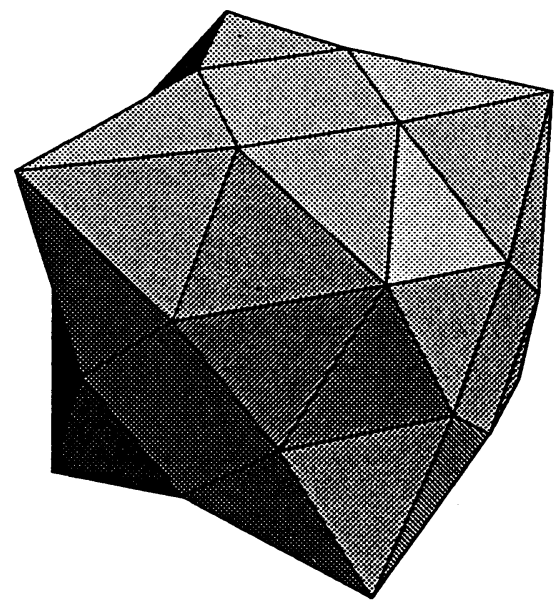

FIGURE 14

$$
\begin{aligned}
V=\frac{1}{3} \sqrt{2}(2 x+2 y)^{3}-\sqrt{2} y^{3} \\
+\frac{8}{3} \sqrt{3}\left(x^{2}+2 x y+\frac{1}{4} y^{2}\right)\left(\frac{1}{4}-\frac{1}{3}(x+y)^{2}\right)^{\frac{1}{2}} .
\end{aligned}
$$

Now $V^{\prime}(x)=0$ implies the 6 -th degree equation

$$
\begin{aligned}
& (236160 \sqrt{2}-334080) x^{6}+(554624-391808 \sqrt{2}) x^{5} \\
& +(235968 \sqrt{2}-334288) x^{4}+(88288-62400 \sqrt{2}) x^{3} \\
& +(6280 \sqrt{2}-8776) x^{2}+(104 \sqrt{2}-160) x+55-40 \sqrt{2}=0 .
\end{aligned}
$$

The relevant zero is $x_{0}=0.2188616575649390 \ldots$. We have $V\left(x_{0}\right)=$ $1.21865263 \ldots$. Since $V(0)=1$, the maximum gain in volume is about $21.9 \%$.

To produce the deformation for the dodecahedron with unit edge length, we start with a regular icosahedron of edge length $2 x+2 y$, where $y=\cos \left(\frac{\pi}{5}\right)-2 \sin \left(\frac{\pi}{5}\right) x$ and $0 \leq x \leq \frac{1}{2} \cot \left(\frac{\pi}{5}\right)$. Again, as in Figure 15, we attach hexagonal pyramids to these faces, so that the distance from apex of any pyramid to a nearest edge of the icosahedron is $\frac{1}{2}$. We truncate the icosahedron, as in Figure 16, so that the 6 new pentagonal faces have edge length $y$. The 20 original faces are truncated to hexagons of alternating edge lengths $2 x$ and $y$. Since $y=\cos \left(\frac{\pi}{5}\right)-2 \sin \left(\frac{\pi}{5}\right) x$, the bounding surface of the resulting solid is intrinsically isometric to the surface of the dodecahedron with unit edge length. The volume of the solid is the volume of the truncated icosahedron plus the total volume 


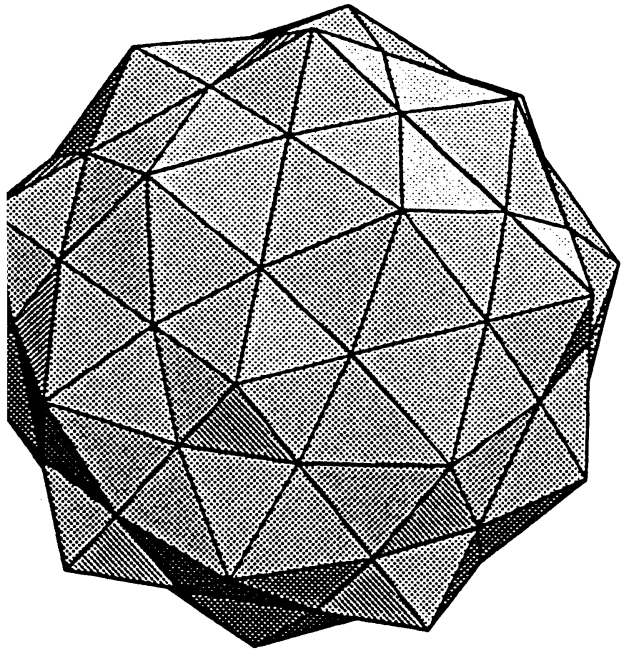

FIGURE 15

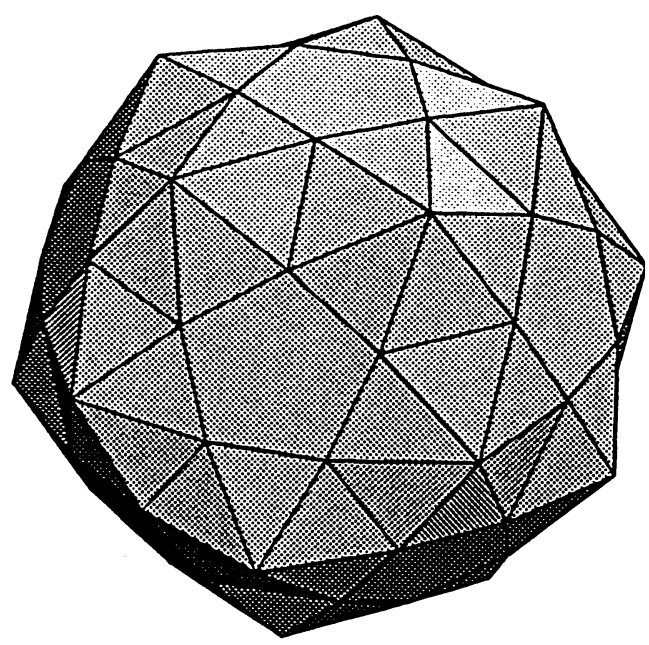

FiguRE 16

of the 20 hexagonal pyramids, namely

$$
\begin{aligned}
V= & \frac{5}{12}(3+\sqrt{5})(2 x+2 y)^{3}-\frac{1}{2}(5+\sqrt{5}) y^{3} \\
& +\frac{20}{3} \sqrt{3}\left(x^{2}+2 x y+\frac{1}{4} y^{2}\right)\left(\frac{1}{4}-\frac{1}{3}(x+y)^{2}\right)^{\frac{1}{2}} .
\end{aligned}
$$

The only relevant zero of the cumbersome sixth degree equation implied by $V^{\prime}(x)=0$ is $x_{0}=0.2863889425606750 \ldots$. From $V\left(x_{0}\right)=$ 8.37883569 and $V(0)=\frac{1}{4}(15+7 \sqrt{5})$, we obtain $V\left(x_{0}\right) / V(0)$ $=1.09339757 \ldots$; i.e., about a $9.3 \%$ increase in volume.

\section{Summary of the regular special cases}

The solids enclosed by the deformations of the surfaces $S$ bounding the platonic solids are each composed of a truncation of a dual solid $D$ and a number of pyramids attached to the truncations, say $F_{i}$, of the original faces of $D$. The newly formed regular faces of the truncation of $D$, we will denote by $G_{j}$. In each case, the bases $F_{i}$ of the pyramids are not regular polygons for the solid of maximal volume in the family of deformations. For the solid of maximal volume, the irregularity of the $F_{i}$ is measured by the deviation of $2 x_{0} / y_{0}$ from 1 , where $2 x_{0}$ is the length of the segment joining a pair of closest vertices of a pair of neighboring $G_{j} s$, and $y_{0}$ is the length of a side of any $G_{j}$. Always, $y_{0}=\cos \left(\frac{\pi}{n}\right)-2 \sin \left(\frac{\pi}{n}\right) x_{0}$, where $n$ is the number of sides per face of the original surface $S$. All of the results obtained are tabulated below. The results for $x_{0}$ (and $2 x_{0} / y_{0}$ ) are sensitive to the value of $n$, but when 
$n=3$ these results are remarkably insensitive to the number of faces of $S$.

\begin{tabular}{|c|c|c|c|}
\hline Polyhedron & $x_{0}$ & $2 x_{0} / y_{0}$ & $V\left(x_{0}\right) / V(0)$ \\
\hline double covered triangle & $0.1354446662193181 \ldots$ & $1.02067187 \ldots$ & $\infty$ \\
\hline tetrahedron & $0.1354198180198074 \ldots$ & $1.02031916 \ldots$ & $1.37718257 \ldots$ \\
\hline cube & $0.2188616575649390 \ldots$ & $1.10094241 \ldots$ & $1.21865263 \ldots$ \\
\hline octahedron & $0.1354140138298575 \ldots$ & $1.02023679 \ldots$ & $1.11580800 \ldots$ \\
\hline dodecahedron & $0.2863889425606750 \ldots$ & $1.21262201 \ldots$ & $1.09339757 \ldots$ \\
\hline icosahedron & $0.1354116696972618 \ldots$ & $1.02020352 \ldots$ & $1.03631161 \ldots$ \\
\hline
\end{tabular}

\section{Some open questions and problems}

1. We have seen that it is possible to isometrically embed a doublecovered equilateral triangle in $E^{3}$. The proof of Theorem 1 shows this for any triangle. Is it possible to isometrically embed any double-covered planar region in $E^{3}$ ? If one can translate Russian, one can ascertain whether the case of polygonal regions was in fact entirely disposed of in [5]. Indeed, this paper reportedly has a proof that any compact, orientable, abstract polyhedral surface (in particular a flat torus) admits a piecewise linear isometric embedding in $E^{3}$. For double-covered planar regions with smooth boundary very little is known, but the present author has shown (unpublished) that the double-covered disk can be isometrically embedded in $E^{3}$.

2. Although not every compact $C^{2}$ Riemannian surface can be $C^{2}$ isometrically embedded in $E^{3}$, there is a nice result due to Kuiper and Nash [12] which states that any compact, orientable, $C^{1}$ Riemannian surface can be $C^{1}$ isometrically embedded in $E^{3}$. One might try to prove that the enclosed volume functional achieves its supremum on the (nonempty) set of all $C^{1}$ isometric embeddings (or immersions) of a given compact, orientable, abstract $C^{1}$ Riemannian surface. R. Connelly (private communication) has obtained some positive results in this direction, in his work on the bellows conjecture (see [8]). Both E. Calabi and F. J. Almgren, Jr. have pointed out to the author that a sequence of isometric embeddings with enclosed volumes tending to the supremum of the enclosed volume functional might not converge to an isometric embedding. W. H. Paulsen's article [14] contains an instructive example 
of this. In [14], it is argued that the limiting shape of a mylar balloon (double-covered disk) when it is fully inflated is a smooth ovaloid which is flat only at the poles (originally the centers of the two disks). On the way to the limit, the surface of the balloon will experience wrinkling and crimping which is responsible for the shortness of the parallels (circular cross sections) of the ovaloid compared to their original lengths in the disks. Paulsen computes the precise shape of the ovaloid and the area decreasing factor (due to the shrinkage of the parallels), which he calls the crimping factor. This example suggests that one will not much have success looking for isometric embeddings which maximize volume, but rather one ought to look for a maximizer on the set of mappings which do not increase lengths of curves. There is some strong evidence that if one has such a maximizer then there is an arbitrarily close $C^{0}$ approximation to the maximizer by an isometric mapping. For example, in [12] there is a proof that a $C^{1}$ embedding of a compact $C^{1}$ Riemannian nmanifold in $E^{n+1}$ which is "short" in the sense that the induced metric is strictly less than the original metric can be $C^{0}$ approximated by a $C^{1}$ isometric embedding. While the enclosed volume maximizer (if any) is most likely "nowhere long", rather than strictly short, by shrinking the map slightly, there is hope that an isometric embedding enclosing approximately the same volume is not far away in the $C^{0}$ sense.

3. What should we call the "nowhere long" mappings (or their images) of an abstract Riemannian n-manifold into $E^{n+1}$ (or some other Riemannian $(\mathrm{n}+1)$-manifold) which maximize the "enclosed volume" functional? The author was thinking that "sandbag surfaces" (or simply "sandbags") might be appropriate, since one can imagine that they are as full of sand as possible, and sandbags do not stretch much. The term "maximal surfaces" is misleading. Also, to include the abstract polyhedral surfaces, one should not restrict the nowhere long mappings to the $C^{1}$ category, but rather allow continuous mappings which preserve rectifiability of curves and which do not increase lengths of curves (e.g., Lipschitz embeddings with Lipschitz constant 1). One can also introduce the notion of a "weak sandbag" whose enclosed volume does not increase under any continuous deformation through nowhere long mappings. Are there weak sandbags which are not sandbags? One can also consider infinitesimal nowhere long deformations, just as infinitesimal isometric deformations of various orders have been considered in the past [10], and hence one has the notion of infinitesimally weak sandbags of various orders. In general, various restrictions on the class of nowhere long embeddings or deformations lead to possibly different types of sandbags. 
4. If possible, show that the regular polyhedral surfaces can be isometrically deformed to enclose more volume than was obtained here. More generally, show that any compact polyhedral hypersurface can be isometrically deformed to enclose greater volume (i.e., there are no polyhedral sandbags). It is quite likely that this can be done, in view of some recent progress the author has recently made in the smooth case as the following theorem indicates.

Theorem 2. (cf. [4]). Let $F: M \rightarrow E^{n+1}$ be a compact, embedded, $C^{\infty}$ hypersurface in $E^{n+1}$, and let $\rho>0$ be the function on $M$ which assigns to each point $x \in M$ the value of the reciprocal of the largest positive normal (or principal) curvature at the point $x$. If none of the normal curvatures of $M$ at $x$ are positive [as in a planar or inwardly dented region], then $\rho(x)$ is defined to be $+\infty$. Let $V_{M}$ be the volume in $E^{n+1}$ enclosed by $M$, and let $\nu_{M}$ be the volume $n$-form on $M$. If we have $\int_{M} \rho \nu_{M}>(n+1) V_{M}$, then $M$ can be continuously deformed through $C^{1}$ isometric embeddings $F(t): M \rightarrow E^{n+1}(0 \leq t<T$, with $F(0)=F)$ such that the enclosed volume $V(t)$ is a strictly increasing function of $t$.

In other words, if a smooth compact hypersurface is sufficiently close to being planar in an average sense (or inwardly dented anywhere), then it cannot be a sandbag. As a corollary, any $C^{\infty}$ embedded hypersurface which is planar (or inwardly dented) on an open subset cannot be a sandbag. Since polyhedral hypersurfaces are largely planar, this result suggests that any polyhedral hypersurface can be isometrically deformed to enclose more volume. More precisely, one can $C^{0}$ approximate any polyhedral hypersurface by a $C^{\infty}$ hypersurface which is planar away from the $(n-1)$-skeleton, and the $C^{\infty}$ approximation can be isometrically deformed to greater volume. Moreover, an examination of the deformation in [4] which is used in proving Theorem 2, reveals that the volume gains do not diminish with the closeness of the $C^{0}$ approximation. However, it is not clear that the deformation can be made piecewise linear in the limit. Thus, the results of the current paper are not subsumed by Theorem 2, and the general question of whether any compact, polyhedral hypersurface can be isometrically deformed to larger enclosed volume (piecewise linearly or not) remains open, but quite plausible.

5. If the supremum in item 2 is achieved by some nowhere long mapping (sandbag), is the image unique up to a motion of the ambient space? What is the nature of the singular set of a sandbag?

6. Are there simple necessary and/or sufficient conditions which guarantee that the boundary of a convex body is a sandbag? Background 
material may be found in [2], [3], [6] and [15]. Using Theorem 2 it is proved in [4], that an ordinary quadratic oblate spheroid in $E^{3}$ with axis ratio greater than $\sqrt{8 / 3}$ is not a sandbag, or even an infinitesimal sandbag. Thus, there are analytic, strictly convex hypersurfaces in $E^{3}$ which are not sandbags. By the isoperimetric inequality, a standard sphere is a sandbag. However, the author has not yet met R. Connelly's challenge to conclusively prove that there are sandbags other than standard spheres. Paulsen's inflated mylar balloon is a very likely candidate, but his demonstration assumes rotational symmetry, and the existence of a regular solution. One might begin by trying to prove that any prolate spheroid is a sandbag, or at least an infinitesimal sandbag (i.e., by perturbing via sufficiently smooth vector fields, one cannot increase the enclosed volume without increasing the metric somewhere).

7. If possible, generalize the results here to higher dimensions and ambient spaces of nonzero constant sectional curvature. For this, note that Theorem 2 above holds in higher dimensions, and one may wish to consult [9], [11] and [15].

\section{References}

[1] A. D. Aleksandrov, Konvexe polyeder, Akademic - Verlag, Berlin, 1958.

[2] A. D. Aleksandrov \& V. A. Zalgaller, Intrinsic geometry of surfaces, (Transl. Math. Monographs, Vol. 15) Providence, RI, Amer. Math. Soc., 1967.

[3] M. Berger, Geometry II, Springer, Berlin, 1987.

[4] D. D. Bleecker, Isometric deformations of compact hypersurfaces, Geom. Dedicata, to appear.

[5] Y. D. Burago \& V. A. Zalgaller, Polyhedral embedding of a net, Vestnik Leningrad. Univ. 15 (1960) 66-80.

[6] H. Busemann, Convex surfaces, Interscience, New York, 1958.

[7] A. Cauchy, Sur les polygones et polyedres, 2nd Mem. J. École Polytechn. 9 (1813) 87.

[8] R. Connelly, Conjectures and open questions in rigidity, Proc. Internat. Congr. Math. (Helsinki, 1978), Acad. Sci., Fennica, Helsinki, 1980.

[9] H. S. M. Coxeter, Regular complex polytopes, Cambridge University Press, Cambridge, 1991.

[10] N. V. Efimov, Qualitative problems in the theory of deformations of surfaces, Differential Geometry and Calculus of Variations, 274-423, Transl., Ser. 1, Vol. 6, Amer. Math. Soc., Providence, RI, 1962.

[11] B. Grünbaum, Convex polytopes, Interscience, New York, 1967.

[12] N. H. Kuiper, On $C^{1}$ isometric imbeddings I, Indag. Math. 17 (1955) $545-556$.

[13] L. A. Lyusternik, Convex figures and polyhedra, Heath, Boston, 1966. 
[14] W. H. Paulsen, What is the shape of a mylar balloon?, Amer. Math. Monthly 101 (1994) 953-958.

[15] A. V. Pogorelov, Extrinsic geometry of convex surfaces, (Translations of Mathematical Monographs, Vol. 35) Amer. Math. Soc., Providence, RI, 1973.

UNIVERSITY OF HAWAII 